\section{BMJ Open} Ophthalmology

\title{
Clinically meaningful visual improvements and predictors of early vision gains with ranibizumab for diabetic macular oedema
}

\author{
Lawrence Morse (D) , ${ }^{1}$ Linda Yau, ${ }^{2}$ Lisa Tuomi ${ }^{2}$
}

To cite: Morse L, Yau L, Tuomi L. Clinically meaningful visual improvements and predictors of early vision gains with ranibizumab for diabetic macular oedema. BMJ Open Ophthalmology 2019;4:e000335. doi:10.1136/ bmjophth-2019-000335

Data reported herein were presented in part at the Association for Research in Vision and Ophthalmology 2013 Annual Meeting, May 2013, Seattle, Washington, USA.

Received 14 May 2019 Revised 10 September 2019 Accepted 21 September 2019

A Check for updates

(c) Author(s) (or their employer(s)) 2019. Re-use permitted under CC BY-NC. No commercial re-use. See rights and permissions. Published by BMJ.

${ }^{1}$ Department of Ophthalmology and Vision Science, University of California, Davis, Sacramento, California, USA

${ }^{2}$ Genentech, Inc, South San Francisco, California, USA

Correspondence to Dr Lawrence Morse; Ismorse@ ucdavis.edu

\section{ABSTRACT}

Objective To determine the time to first clinically meaningful improvement in best-corrected visual acuity (BCVA) in patients treated with ranibizumab for diabetic macular oedema (DME) and identify predictors of early visual improvement.

Methods and analysis We retrospectively analysed the phase III RIDE (NCT00473382) and RISE (NCT00473330) trials, in which 759 patients with DME were randomised to monthly intravitreal ranibizumab $0.3 \mathrm{mg}(\mathrm{n}=250)$, ranibizumab $0.5 \mathrm{mg}(\mathrm{n}=252)$ or sham treatment $(\mathrm{n}=257)$. After month 24, 191 sham-treated patients crossed over to monthly ranibizumab $0.5 \mathrm{mg}$ through month 36 , while ranibizumab-treated patients continued treatment. KaplanMeier analyses assessed time to achieve $\geq 15$ or $\geq 10$ Early Treatment Diabetic Retinopathy Study (ETDRS) letter gains from baseline or $\geq 20 / 40$ Snellen equivalent BCVA in each treatment arm. Baseline predictors of $\geq 15$ ETDRS letter gains at month 6 were identified by logistic regression. Results Median time to first $\geq 15$ ETDRS letter gain was significantly shorter in patients who received ranibizumab (0.3 mg, 11.1 months; $0.5 \mathrm{mg}, 10.9$ months) than shamtreated patients who crossed over to ranibizumab $0.5 \mathrm{mg}$ at month 24 (35.7 months; both $p<0.0001)$. Half of ranibizumab-treated patients achieved $\geq 20 / 40$ BCVA within $2.3(0.3 \mathrm{mg})$ and 1.9 months $(0.5 \mathrm{mg})$. Baseline predictors of early vision improvement among ranibizumab-treated patients were BCVA $\leq 55$ ETDRS letters, younger age and presence of subretinal fluid.

Conclusion Prompt ranibizumab therapy for DME was associated with rapid, clinically meaningful vision gains that were maintained over 36 months of treatment. Lower BCVA, younger age and presence of subretinal fluid were predictive of early vision improvement.

\section{INTRODUCTION}

Diabetic retinopathy (DR) is a leading cause of blindness among working-age adults in the developed world and is estimated to affect $35 \%$ of individuals with diabetes aged 20-79 years. ${ }^{1}$ Diabetic macular oedema (DME), a vision-threatening complication of DR, develops in $\sim 20 \%-30 \%$ of individuals with a $\geq 20$-year duration of diabetes. ${ }^{12}$ Although the pathophysiology of DME is not fully understood, chronic hyperglycaemia is believed

\section{Key messages}

What is already known about this subject?

> The phase III RIDE and RISE trials have previously demonstrated the safety and efficacy of intravitreal ranibizumab in patients with diabetic macular oedema (DME). To better understand functional responses to ranibizumab, this posthoc analysis quantified the time to achieve clinically meaningful visual improvements in RIDE/RISE and identified patient characteristics that were predictive of early vision gains.

\section{What are the new findings?}

$>$ On average, patients randomised to receive ranibizumab at RIDE/RISE baseline first achieved $20 / 40$ or better vision after 3 monthly injections and gained $\geq 15$ Early Treatment Diabetic Retinopathy Study (ETDRS) letters in best-corrected visual acuity (BCVA) within 1 year of treatment. In these patients, clinically meaningful visual improvements were maintained over 36 months of treatment and occurred earlier than in sham-treated patients who received delayed ranibizumab therapy after month 24 . Patient factors associated with an early visual response to ranibizumab (defined as a BCVA gain of $\geq 15$ ETDRS letters from baseline at month 6 ) included younger age, lower BCVA and the presence of subretinal fluid at baseline.

\section{How might these results change the focus of research or clinical practice? \\ These data highlight the value of prompt versus delayed ranibizumab therapy for DME and may be used to manage patient expectations and inform treatment decisions in clinical practice.}

to cause retinal microvascular damage and increased expression of vascular endothelial growth factor (VEGF). ${ }^{3}$ Along with pathological angiogenesis, VEGF upregulation promotes increased vascular permeability, contributing to the breakdown of the bloodretina barrier and the vascular leakage underlying DME. ${ }^{3}$

Intravitreal ranibizumab, a monoclonal antibody fragment targeted against VEGF-A, 
has been shown to decrease macular oedema and improve vision in patients with $\mathrm{DME}^{4-7}$ and is approved for this indication in several regions, including Europe ${ }^{8}$ and the USA. ${ }^{9}$ In particular, evidence that informed the US Food and Drug Administration approval of ranibizumab for DME was primarily derived from RIDE and RISE, two methodologically identical, phase III, multicentre, double-masked, randomised controlled trials that evaluated the efficacy and safety of intravitreal ranibizumab in patients with vision loss due to DME. ${ }^{4}$ Compared with those who received sham injections, a significantly greater proportion of ranibizumab-treated patients in RIDE and RISE gained $\geq 15$ Early Treatment Diabetic Retinopathy Study (ETDRS) letters in best-corrected visual acuity (BCVA) from baseline to 24 months; ${ }^{4}$ BCVA gains achieved during this period were maintained through month 36 with ongoing ranibizumab treatment. ${ }^{5}$

At month 24, sham patients in RIDE and RISE crossed over to receive monthly ranibizumab $0.5 \mathrm{mg}$, which allowed investigators to assess the effect of delayed treatment on visual outcomes. ${ }^{5}$ Vision gains observed in the sham crossover group between months 24 and 36 were lower than that achieved by the ranibizumab treatment arm from baseline to 12 months, ${ }^{5}$ suggesting that prompt intervention may be necessary to avoid the irreversible consequences of chronic DME and maximise patient responses to therapy.

Understanding patterns of response to ranibizumab and predictors of early visual improvement may inform physician and patient expectations of ranibizumab therapy in clinical practice. Using data obtained from RIDE and RISE, this retrospective analysis aimed to quantify the time to clinically meaningful vision gains in patients with DME treated with ranibizumab and to identify baseline patient and ocular characteristics that predict early responses to treatment.

\section{MATERIALS AND METHODS}

\section{RIDE and RISE}

Full details of the study design, patient population, methods and primary outcomes of RIDE and RISE are reported elsewhere. ${ }^{45}$ Briefly, RIDE (ClinicalTrials.gov identifier NCT00473382) and RISE (NCT00473330) included patients aged $\geq 18$ years, with diabetes mellitus (type 1 or 2), vision loss due to DME (BCVA 20/4020/320 Snellen equivalent) and macular oedema identified by time-domain optical coherence tomography. One eye per patient was randomised to receive monthly intravitreal injections of ranibizumab $0.3 \mathrm{mg}$, ranibizumab $0.5 \mathrm{mg}$ or sham treatment; after month 24, sham patients were eligible to receive monthly treatment with ranibizumab $0.5 \mathrm{mg}$ for the remainder of the 36-month treatment period. From month 3 onwards, all patients were eligible to receive macular laser therapy according to protocol-specified criteria; panretinal laser was available as clinically indicated.

The primary efficacy endpoint of RIDE and RISE was the proportion of patients who gained $\geq 15$ ETDRS letters in BCVA from baseline at month $24 .{ }^{4}$ Secondary endpoints relevant to the present analysis included mean change from baseline BCVA over time and the proportion of patients who achieved BCVA of 20/40 or better (equivalent to 70 ETDRS letters). Both trials were conducted in accordance with the Declaration of Helsinki and were compliant with the Health Insurance Portability and Accountability Act. Protocols were approved by institutional review boards, ethics committees or as applicable, and all patients provided written informed consent.

\section{Posthoc analyses}

Data from RIDE and RISE were pooled in the present study, with analyses conducted on the intention-to-treat population by treatment arm. In the analyses of primary and secondary endpoints, missing BCVA values were imputed using last observation carried forward methodology. In the posthoc analyses described below, missing BCVA values were not imputed. Patient and public involvement was not sought for the design, conduct and reporting of this study.

\section{Time to clinically meaningful visual improvement}

Kaplan-Meier analyses were conducted to estimate the cumulative proportion of patients who achieved clinically meaningful visual improvements by month 36 and the time to first occurrence of such an event. Clinically meaningful visual improvements were defined as a gain of $\geq 15$ or $\geq 10$ ETDRS letters from baseline or the achievement of BCVA 20/40 or better. Time-to-event distributions for each of these definitions were compared between treatment arms using two-sided log-rank tests, with a threshold probability value of $\mathrm{p} \leq 0.05$ selected $a$ priori to indicate statistical significance. Patients who did not achieve a clinically meaningful visual improvement by month 36 were censored at the last available BCVA assessment.

\section{Predictors of early visual improvement}

Factors predictive of early visual improvement, defined as a BCVA gain of $\geq 15$ ETDRS letters from baseline at month 6 , were identified by multiple logistic regression modelling. Candidate covariates included baseline patient characteristics, demographics and targeted medical histories (table 1); significant variables were selected by the stepwise selection algorithm in models fitted separately for each treatment arm. A final model derived for the overall cohort included significant variables identified from each of the separate models, in addition to a treatment variable (ranibizumab-treated versus sham) and appropriate interaction terms. Odds ratios (ORs) and 95\% CIs were estimated for each factor from the final logistic regression model.

\section{RESULTS}

As previously described, ${ }^{45} 759$ patients with DME were initially randomised to receive monthly sham injections $(\mathrm{n}=257)$, ranibizumab $0.3 \mathrm{mg}(\mathrm{n}=250)$ or ranibizumab 
Table 1 Potential predictors of early visual improvement in RIDE and RISE

\section{Baseline patient characteristics/demographics}

\section{Age}

Race and ethnicity

Sex

Current smoker

Oedema type (focal/diffuse/absent, NA or unknown)

Prior DME therapy

BCVA ( $\leq 55$ vs $>55$ ETDRS letters)

Proliferative diabetic retinopathy

Central foveal thickness

Presence of subretinal fluid

Chronic kidney disease (eGFR $<60 \mathrm{~mL} / \mathrm{min} / 1.73 \mathrm{~m}^{2}$ )

Duration of diabetes at randomisation

$\mathrm{HbA1c}$

\section{Bilateral DME}

Insulin use

Systolic blood pressure

Diastolic blood pressure

Intraocular pressure

BCVA, best-corrected visual acuity; DME, diabetic macular oedema; eGFR, estimated glomerular filtration rate; ETDRS, Early Treatment

Diabetic Retinopathy Study; HbA1c, glycated haemoglobin; NA, not applicable.

$0.5 \mathrm{mg}(\mathrm{n}=252)$. After 24 months, 191 of 210 sham patients who remained in the study crossed over to active treatment and received monthly ranibizumab $0.5 \mathrm{mg}$ through month 36 (sham $/ 0.5 \mathrm{mg}$ group). Patient demographics and study eye characteristics were well balanced across treatment arms at baseline. ${ }^{4}$ Over 36 months, a greater proportion of sham-treated patients received macular or panretinal laser therapy to the study eye compared with patients who received ranibizumab (table 2 ).

\section{Patients with clinically meaningful visual improvement}

The proportion of patients who gained $\geq 15$ ETDRS letters in BCVA increased rapidly among those randomised to receive ranibizumab and was consistently greater than the sham-treated group over 36 months (figure 1A). At month 6, 27.2\% and $23.8 \%$ of patients treated with ranibizumab $0.3 \mathrm{mg}$ and $0.5 \mathrm{mg}$ gained $\geq 15$ ETDRS letters from baseline, respectively, compared with $8.6 \%$ of sham-treated patients. Following crossover at month 24 , the proportion of sham $/ 0.5 \mathrm{mg}$ patients

\section{Targeted medical histories}

Hypertension

Proteinuria

Rheumatoid arthritis

Any neovascular condition

Any cardiovascular condition

Glaucoma in study eye

Dermatitis

Any clotting/bleeding condition

Renal failure

Diverticular disease who gained $\geq 15$ ETDRS letters from baseline increased modestly to $20.6 \%$ at month 36 , but remained lower than the ranibizumab $0.3 \mathrm{mg}$ and $0.5 \mathrm{mg}$ treatment arms $(44.0 \%$ and $40.9 \%$, respectively; figure $1 \mathrm{~B})$.

Similarly, a greater proportion of patients randomised to ranibizumab at RIDE/RISE baseline achieved BCVA $20 / 40$ or better at month 36 , compared with sham crossover patients (figure 1C). At baseline, $19.5 \%$ of sham $/ 0.5 \mathrm{mg}$ patients, $16.4 \%$ of ranibizumab $0.3 \mathrm{mg}$ patients and $19.4 \%$ of ranibizumab $0.5 \mathrm{mg}$ patients had $20 / 40$ or better vision; these proportions increased to $42.4 \%, 59.2 \%$ and $59.1 \%$, respectively, at month 36 . Among those who achieved BCVA 20/40 or better at month $36(n=406), 29.1 \%$ had this level of vision at baseline.

Time to clinically meaningful visual improvement Clinically meaningful visual improvements were achieved earlier in ranibizumab-treated than sham-treated patients in RIDE and RISE, irrespective of the endpoint used to define such an event (figure 2). As shown in figure 2A,

Table 2 Treatments administered to study eyes through month 36 of RIDE and RISE, stratified by treatment arm (pooled data)

\begin{tabular}{lccc}
\hline Treatment & Sham $(\mathbf{n}=\mathbf{2 5 7})$ & Ranibizumab 0.3 $\mathbf{m g}(\mathbf{n}=\mathbf{2 5 0})$ & Ranibizumab 0.5 mg $(\mathbf{n}=\mathbf{2 5 2})$ \\
\hline Median number of ranibizumab injections & 11 & 34 & 34 \\
Macular laser treatment, $\mathrm{n}(\%)$ & $188(73.2)$ & $97(38.8)$ & $74(29.4)$ \\
Panretinal laser treatment, $\mathrm{n}(\%)$ & $34(13.2)$ & $4(1.6)$ & $6(2.4)$
\end{tabular}


A

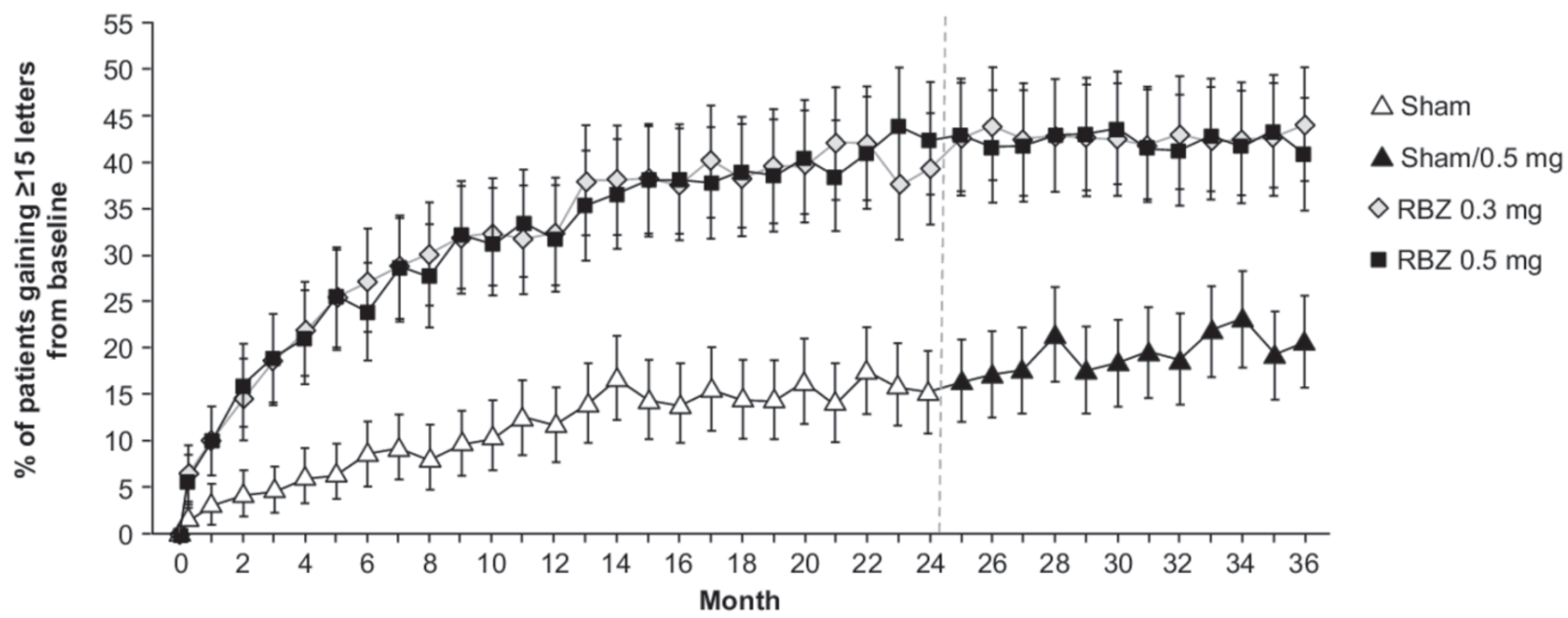

B

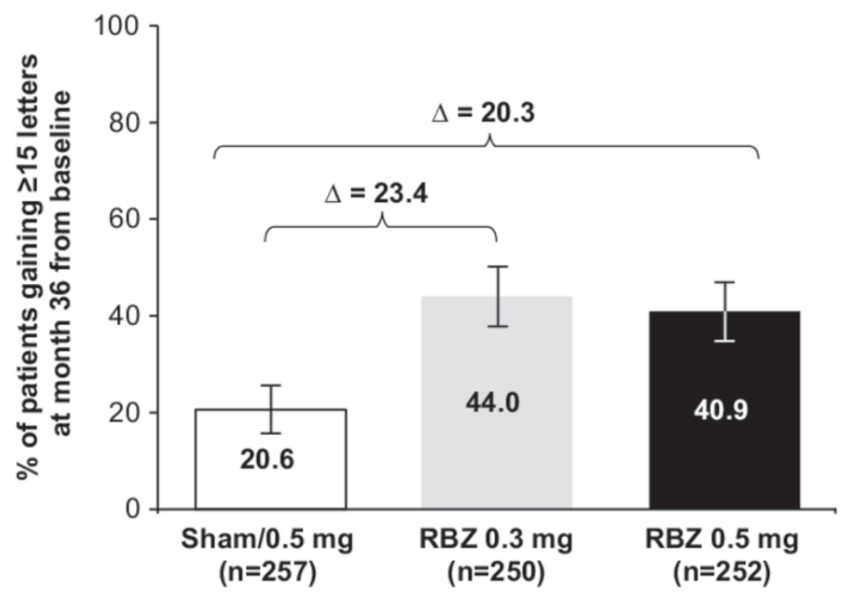

C

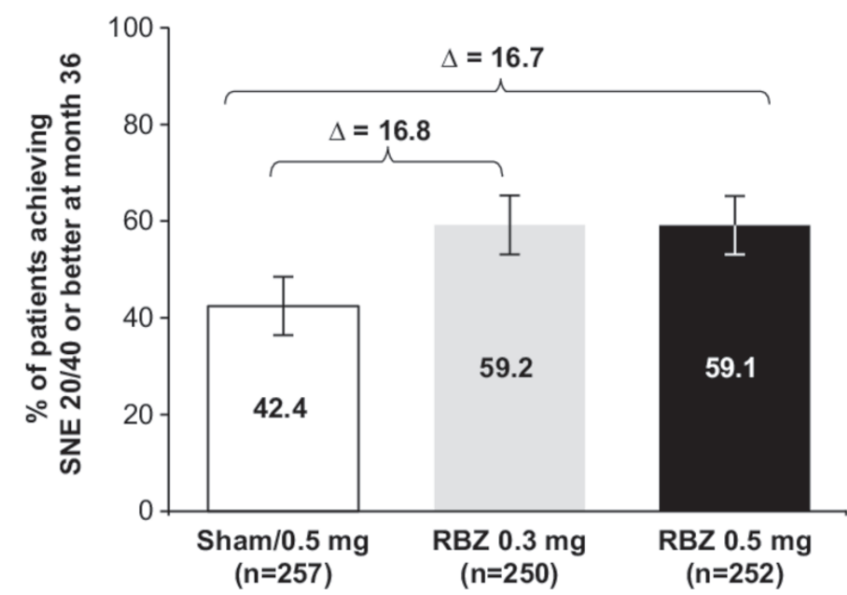

Figure 1 Proportion of RIDE/RISE patients with BCVA gains of $\geq 15$ Early Treatment Diabetic Retinopathy Study letters from baseline (A) over time and (B) at month 36; (C) proportion of RIDE/RISE patients who achieved BCVA 20/40 or better SNE at month 36 . Error bars represent $95 \%$ Cls; missing values were imputed by last observation carried forward. BCVA, bestcorrected visual acuity; RBZ, ranibizumab; SNE, Snellen equivalent.

the median time to first gain of $\geq 15$ ETDRS letters from baseline was significantly shorter in the ranibizumab $0.3 \mathrm{mg}$ and $0.5 \mathrm{mg}$ treatment arms (11.1 months and 10.9 months, respectively) compared with the sham $/ 0.5 \mathrm{mg}$ group (35.7 months; both $\mathrm{p}<0.0001$ ) and was not estimable in sham patients who did not cross over to ranibizumab treatment (sham/no crossover group; $\mathrm{n}=66$ ). The cumulative proportions of patients who ever gained $\geq 15$ ETDRS letters by month 36 were $72 \%$ and $71 \%$ in patients treated with ranibizumab $0.3 \mathrm{mg}$ and $0.5 \mathrm{mg}$, respectively, compared with $52 \%$ of sham $/ 0.5 \mathrm{mg}$ patients and $38 \%$ of sham/no crossover patients. In an exploratory time-to-event analysis of vision gains achieved after crossover at month 24, we found that the median time to first gain of $\geq 15$ ETDRS letters from crossover was not estimable in the sham $/ 0.5 \mathrm{mg}$ group (data not shown). Cumulatively, less than $25 \%$ of sham $/ 0.5 \mathrm{mg}$ patients ever achieved a $\geq 15$ ETDRS letter gain from crossover by month 36 ; in contrast, over $50 \%$ of patients randomised to ranibizumab at RIDE/RISE baseline ever gained $\geq 15$ ETDRS letters within the first 12 months of treatment.

Early responses to ranibizumab treatment were also observed when clinically meaningful visual improvements were defined as a gain of $\geq 10$ ETDRS letters from baseline (figure 2B) or the achievement of BCVA 20/40 or better (figure 2C). The median time to first gain of $\geq 10$ ETDRS letters from baseline was significantly earlier in patients treated with ranibizumab $0.3 \mathrm{mg}$ and $0.5 \mathrm{mg}$ than either the sham $/ 0.5 \mathrm{mg}$ or sham/no crossover groups (3.8 months and 2.9 months vs 14.1 months and 29.7 months, respectively; all $\mathrm{p}<0.0001)$. Similarly, the median time to first achievement of BCVA $20 / 40$ or better was significantly shorter in the ranibizumab $0.3 \mathrm{mg}$ and $0.5 \mathrm{mg}$ treatment arms (2.3 months and 1.9 months, respectively) compared with sham $/ 0.5 \mathrm{mg}$ patients $(8.1$ months; $\mathrm{p}=0.002$ and $\mathrm{p}<0.001$, respectively) and sham/ no crossover patients ( 4.7 months; $\mathrm{p}=0.036$ and $\mathrm{p}=0.009$, respectively). 
A
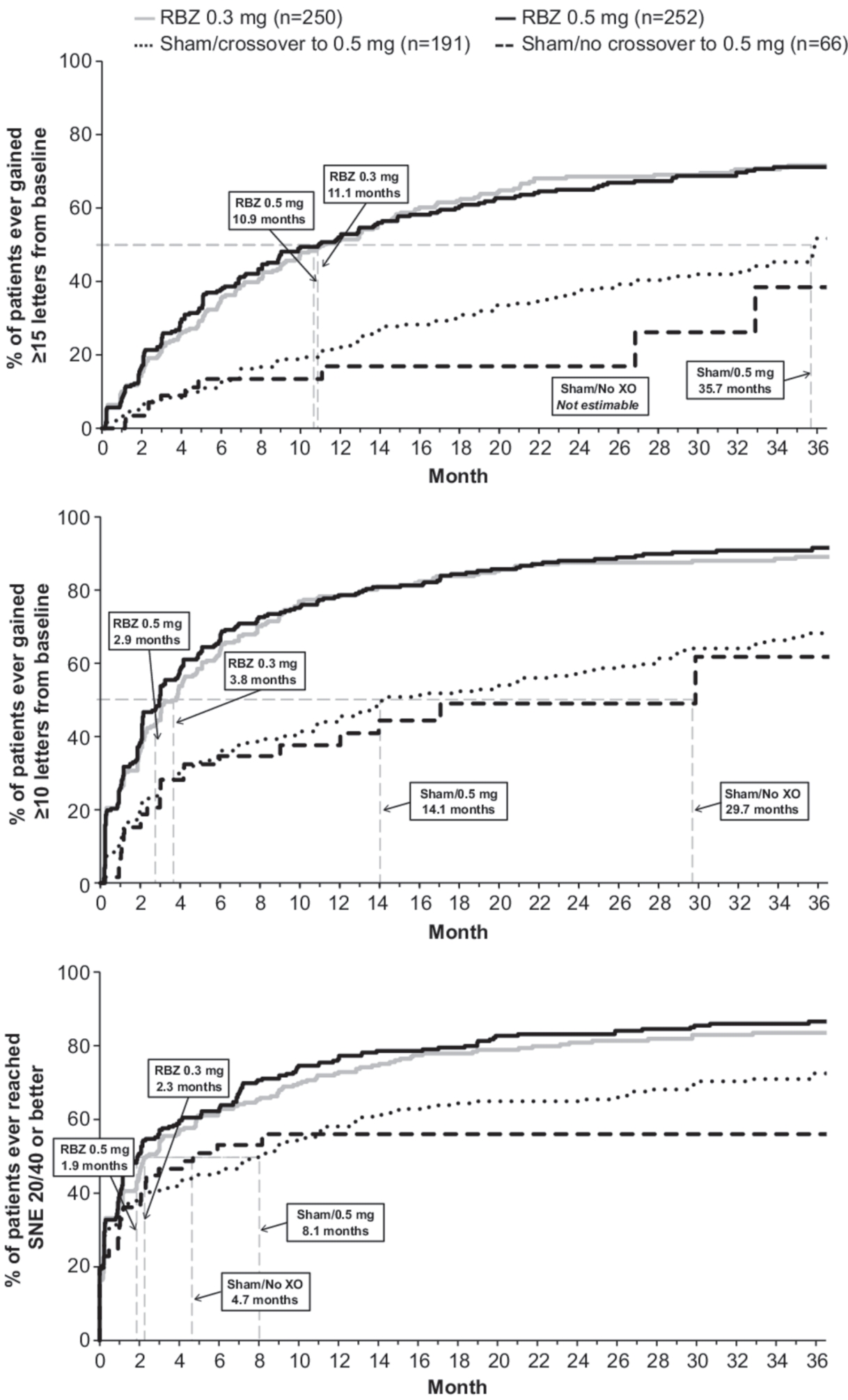

Figure 2 Kaplan-Meier plot of time to first BCVA gain of $(A) \geq 15$ and $(B) \geq 10$ Early Treatment Diabetic Retinopathy Study letters from baseline; (C) Kaplan-Meier plot of time to first achievement of BCVA 20/40 or better SNE. RIDE/RISE pooled data; missing values were not imputed. BCVA, best-corrected visual acuity; RBZ, ranibizumab; SNE, Snellen equivalent; XO, crossover.

Predictors of early visual improvement

Factors predictive of early visual improvement, defined as a BCVA gain of $\geq 15$ ETDRS letters from baseline at month 6 , are presented in figure 3 . Of the characteristics examined, baseline BCVA of $\leq 55$ ETDRS letters (OR 3.84,
95\% CI 2.47 to 5.98) and younger baseline age (OR per 10 -year decrease $1.65,95 \%$ CI 1.33 to 2.04 ) were associated with greater likelihood of early visual improvement across all patients, while the presence of subretinal fluid at baseline was predictive of early visual improvement in 


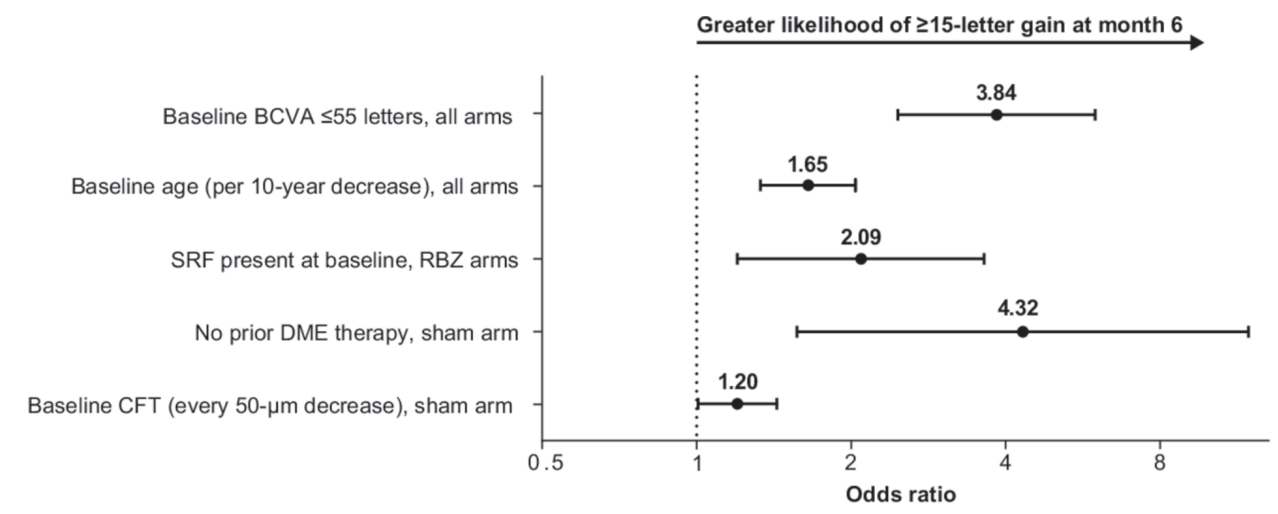

Figure 3 Factors predictive of early visual improvement in RIDE/RISE, defined as a BCVA gain of $\geq 15$ Early Treatment Diabetic Retinopathy Study letters from baseline at month 6. Error bars represent $95 \% \mathrm{Cls}$; missing values were not imputed in logistic regression analyses. BCVA, best-corrected visual acuity; CFT, central foveal thickness; DME, diabetic macular oedema; RBZ, ranibizumab; SRF, subretinal fluid.

the ranibizumab treatment arms (OR 2.09, 95\% CI 1.20 to 3.63). Among sham-treated patients, no prior DME therapy (OR 4.32, 95\% CI 1.57 to 11.9) and lower central foveal thickness at baseline (OR per 50- $\mu$ m decrease 1.20, $95 \%$ CI 1.01 to 1.43 ) were associated with greater likelihood of early visual improvement. In additional analyses, similar factors were identified as predictors of a BCVA gain of $\geq 10$ ETDRS letters from baseline at month 6 (data not shown).

\section{DISCUSSION}

RIDE and RISE previously showed that ranibizumab therapy improved visual acuity and anatomic outcomes in patients with DME and that such benefits were maintained over 36 months of treatment. ${ }^{45}$ To further explore patient responses to ranibizumab, this retrospective analysis of RIDE and RISE compared the time to achieve clinically meaningful visual improvements between treatment arms and identified factors that predict early responses to therapy. Compared with sham controls, patients treated with ranibizumab benefited from significantly earlier vision gains, with $>50 \%$ of patients achieving BCVA $20 / 40$ or better within 3 months, gaining $\geq 10$ ETDRS letters within 4 months and/or gaining $\geq 15$ ETDRS letters within 12 months. Baseline patient characteristics associated with an early response to ranibizumab included lower BCVA, younger age and the presence of subretinal fluid. However, the cumulative proportion of ranibizumab-treated patients who ever achieved clinically meaningful visual improvements exceeded $70 \%$ by month 36 , indicating that vision gains are nevertheless achievable with ongoing therapy.

In this study, the proportion of patients who achieved clinically meaningful visual improvements at month 36 was lower among sham-treated patients who switched to ranibizumab therapy after month 24 than those who received ranibizumab $0.3 \mathrm{mg}$ and $0.5 \mathrm{mg}$ from RIDE/ RISE baseline. This finding is consistent with previous research $^{5}$ and suggests that chronic oedema may impact a patient's capacity to gain vision with future treatment. Alternatively, photoreceptor damage caused by macular and/or panretinal laser therapy, which were received by more sham-treated patients in RIDE and RISE, may also limit visual responses to subsequent ranibizumab treatment. RIDE and RISE were not designed to compare laser therapy with ranibizumab; therefore, we were unable to determine whether suboptimal vision gains achieved by sham $/ 0.5 \mathrm{mg}$ patients were the result of laser therapy or delayed ranibizumab treatment. Nevertheless, patients with DME who initiated prompt ranibizumab therapy benefited from rapid vision improvements that were sustained over 36 months, which highlights the importance of early intervention to maximise treatment responses.

The results of our time-to-event analyses suggest that patients with DME may resume activities of daily living soon after initiating monthly ranibizumab therapy. For example, 20/40 vision in one or both eyes is the minimum requirement for a driving licence in many countries ${ }^{10} 11$ and is an acceptable threshold for daily tasks requiring an adequate level of visual acuity. In this study, the median time to BCVA $20 / 40$ or better was $\sim 2$ months in RIDE/ RISE patients who received prompt ranibizumab therapy, which was significantly earlier than in sham $/ 0.5 \mathrm{mg}$ and sham/no crossover patients. More than $80 \%$ of patients randomised to receive ranibizumab $0.3 \mathrm{mg}$ or $0.5 \mathrm{mg}$ in RIDE/RISE achieved 20/40 or better vision at any point over the study period, which was maintained by $\sim 60 \%$ of patients at month 36 .

Identifying factors that predict functional and anatomical responses to ranibizumab may inform and manage treatment expectations among clinicians and patients. Previously, factors associated with an early anatomical response to ranibizumab in RIDE/RISE (defined as an improvement of $\geq 2$ steps on the ETDRS Diabetic Retinopathy Severity Scale ${ }^{12}$ from baseline at month 6) included baseline DR severity (particularly moderately severe to severe non-proliferative DR), no prior panretinal photocoagulation at baseline and the absence of DME at month $3{ }^{13}$ In the present study, baseline characteristics predictive of an early visual response to ranibizumab in RIDE/ RISE were lower BCVA, younger age and the presence 
of subretinal fluid. While a lack of these characteristics may conversely reduce the likelihood of achieving early vision gains with ranibizumab, further studies are needed to identify other patient factors that predict a delayed or suboptimal response to treatment.

Our findings are consistent with other posthoc analyses that identified poor baseline BCVA, subretinal fluid at baseline and young age as predictors of $\geq 15$ ETDRS letter gains from baseline at month $24 .{ }^{14}$ Lower BCVA and younger age at baseline were similarly associated with larger 1-year visual responses to ranibizumab in the Diabetic Retinopathy Clinical Research Network (DRCR. net) Protocol I study, in addition to less severe DR on clinical examination and the absence of surface wrinkling retinopathy. ${ }^{15}$ The presence of proliferative DR was not identified as a predictor of early vision gains in the present study, likely due to the exclusion of patients with active proliferative DR in RIDE and RISE.

It is unclear why younger age may be predictive of early vision gains with ranibizumab therapy; however, it has previously been hypothesised that the macula of younger patients may tolerate fluid accumulation without incurring loss of visual potential. ${ }^{14}$ Our observation that lower baseline BCVA was associated with early treatment response is suggestive of a 'ceiling effect'; in other words, smaller improvements are required to achieve good vision in patients with higher BCVA at baseline, while those with poorer starting vision have greater capacity to achieve clinically meaningful visual gains over the course of treatment. Although further research is required to understand the association between subretinal fluid and early visual improvements, our findings may suggest that subretinal fluid is less disruptive to vision gains than intraretinal fluid or that eyes with subretinal fluid may be more responsive to ranibizumab treatment. Additional analyses of sham-treated patients showed that predictors of early treatment response included a lack of prior DME therapy and reduced central foveal thickness, characteristics consistent with less severe disease at baseline. Vision gains in the sham arm were likely driven by laser therapy, which has been associated with modest benefits in some patients with DME. ${ }^{1617}$

Interestingly, neither baseline glycated haemoglobin (HbA1c) nor other systemic characteristics associated with diabetes were predictive of an early visual response to ranibizumab therapy. Similar findings have been reported in previous analyses of RIDE and RISE, which showed no association between systemic factors (including antihyperglycaemic medication history, serum glucose, renal function, body mass index, blood pressure and $\mathrm{HbA1c}$ ) and functional or anatomic outcomes such as BCVA improvements and reductions in central foveal thickness. ${ }^{18} 19$ At the discretion of their evaluating physician, all patients in RIDE/RISE were permitted to continue standard treatments for diabetes; as a result, HbA1c and blood glucose at baseline and month 24 were similar between ranibizumab-treated and sham-treated patients, and no association was found between these parameters and BCVA gains achieved with ranibizumab therapy. ${ }^{19}$ Taken together, these data suggest that patient responses to ranibizumab are independent of non-ocular factors related to the systemic management of diabetes. However, because most RIDE/RISE patients had HbAlc $\leq 8 \%$ at baseline, patients with uncontrolled diabetes (HbA1c $>12 \%)$ were excluded and follow-up was limited to 3 years, ${ }^{45}$ caution is required when generalising our analyses to alternative populations with elevated and/ or uncontrolled HbAlc and extrapolating results over longer time horizons. It is well established that longterm glycaemic control is imperative to manage the risk of macrovascular and microvascular complications, including DR $;{ }^{20}$ therefore, further studies are required to characterise direct relationships between systemic control of diabetes and patient responses to ranibizumab.

In conclusion, this posthoc analysis of RIDE and RISE demonstrated the value of prompt versus delayed ranibizumab therapy for DME and identified predictors of early treatment responses in this patient population. The majority of patients randomised to receive ranibizumab at RIDE/RISE baseline achieved 20/40 or better vision after 3 monthly injections and gained $\geq 15$ ETDRS letters from baseline within 1 year of treatment. Clinically meaningful visual improvements in these patients were maintained through month 36 and occurred earlier than in sham-treated patients who received delayed ranibizumab treatment at month 24. Baseline predictors of early vision gains among ranibizumab-treated patients included lower BCVA, younger age and the presence of subretinal fluid, information which may be used to manage patient expectations and inform treatment decisions in clinical practice. Data arising from this study provide an indication of how quickly patients with DME may regain vision and resume daily activities with monthly ranibizumab therapy and support the notion of early DME management in conjunction with systemic control of diabetes.

Acknowledgements The authors thank the RIDE/RISE study group and investigators for their contributions to these trials; Jason S. Ehrlich, MD, PhD, of Genentech, Inc. for his contributions to RIDE/RISE and the initiation and concept stages of the present analysis; and Jeffrey R. Willis, MD, PhD, of Genentech, Inc. for his contributions to the manuscript

Contributors LM and LT contributed to the design and conduct of the study and data collection. LM, LY and LT contributed to data analysis, interpretation and critical review and final approval of the manuscript. LM contributed to provision of materials, patients or resources. LY contributed to statistical expertise.

Funding Genentech, Inc., a member of the Roche Group, provided financial support for the study, participated in the design and conduct of the study and was involved in data collection, management and interpretation. Third-party writing assistance, provided by Karina D. Hamilton-Peel, PhD, Jack W. Pike, PhD and Kathryn H. Condon, PhD, of Envision Pharma Group, was funded by Genentech, Inc.

Competing interests LM serves as a consultant for Allergan, Genentech, Inc. and Iridex. LY and LT are employees of Genentech, Inc.

Patient consent for publication Not required.

Provenance and peer review Not commissioned; externally peer reviewed.

Data availability statement Qualified researchers may request access to individual patient level data through the clinical study data request platform ( www.clinicalstudydatarequest.com). Further details on Roche's criteria for eligible 
studies are available here (https://clinicalstudydatarequest.com/Study-Sponsors/ Study-Sponsors-Roche.aspx). For further details on Roche's Global Policy on the Sharing of Clinical Information and how to request access to related clinical study documents, see here (https://www.roche.com/research_and_development/who_ we_are_how_we_work/clinical_trials/our_commitment_to_data_sharing.htm).

Open access This is an open access article distributed in accordance with the Creative Commons Attribution Non Commercial (CC BY-NC 4.0) license, which permits others to distribute, remix, adapt, build upon this work non-commercially, and license their derivative works on different terms, provided the original work is properly cited, appropriate credit is given, any changes made indicated, and the use is non-commercial. See: http://creativecommons.org/licenses/by-nc/4.0/.

ORCID iD

Lawrence Morse http://orcid.org/0000-0002-1758-2348

\section{REFERENCES}

1 Yau JWY, Rogers SL, Kawasaki R, et al. Global prevalence and major risk factors of diabetic retinopathy. Diabetes Care 2012;35:556-64.

2 Klein R, Klein BE, Moss SE, et al. The Wisconsin epidemiologic study of diabetic retinopathy. IV. Diabetic macular edema. Ophthalmology 1984;91:1464-74.

3 Boyer DS, Hopkins JJ, Sorof J, et al. Anti-vascular endothelial growth factor therapy for diabetic macular edema. Ther Adv Endocrinol Metab 2013;4:151-69.

4 Nguyen QD, Brown DM, Marcus DM, et al. Ranibizumab for diabetic macular edema: results from 2 phase III randomized trials: RISE and RIDE. Ophthalmology 2012;119:789-801.

5 Brown DM, Nguyen QD, Marcus DM, et al. Long-term outcomes of ranibizumab therapy for diabetic macular edema: the 36-month results from two phase III trials: RISE and RISE. Ophthalmology 2013;120:2013-22.

6 Mitchell P, Bandello F, Schmidt-Erfurth U, et al. The RESTORE study: ranibizumab monotherapy or combined with laser versus laser monotherapy for diabetic macular edema. Ophthalmology 2011;118:615-25

7 Elman MJ, Aiello LP, Beck RW, et al. Randomized trial evaluating ranibizumab plus prompt or deferred laser or triamcinolone plus prompt laser for diabetic macular edema. Ophthalmology 2010;117:1064-77. e35.

8 European Medicines Agency. Lucentis (ranibizumab), 2018. Available: https://www.ema.europa.eu/en/medicines/human/EPAR/ lucentis [Accessed 19 Feb 2019].
9 Lucentis [package insert]. South San Francisco, CA: Genentech, Inc.; 2018.

10 European Council of Optometry and Optics. Visual standards for driving in Europe. A consensus paper, 2017. Available: https:// www.ecoo.info/wp-content/uploads/2017/01/Visual-Standards-forDriving-in-Europe-Consensus-Paper-January-2017....pdf [Accessed 19 Feb 2019].

11 Carr D, Schwartzberg J, Manning L, et al. Physician's guide to assessing and counseling older drivers. 2nd ed. Washington, DC: National Highway Traffic Safety Administration, 2010.

12 Early Treatment Diabetic Retinopathy Study Research Group. Fundus photographic risk factors for progression of diabetic retinopathy. ETDRS report number 12 . Ophthalmology 1991;98:823-33.

13 Singer MA, Tuomi L, Hill L, et al. Predictors of early improvements in diabetic retinopathy in a significant proportion of patients with 3 or more ranibizumab injections. Presented at: 41 st Annual Macula Society Meeting: 21-24 February, 2018.

14 Sophie R, Lu N, Campochiaro PA. Predictors of functional and anatomic outcomes in patients with diabetic macular edema treated with ranibizumab. Ophthalmology 2015;122:1395-401.

15 Bressler SB, Qin H, Beck RW, et al. Factors associated with changes in visual acuity and central subfield thickness at 1 year after treatment for diabetic macular edema with ranibizumab. Arch Ophthalmol 2012;130:1153-61.

16 Early Treatment Diabetic Retinopathy Study Research Group. Photocoagulation for diabetic macular edema. Early Treatment Diabetic Retinopathy Study report number 1. Arch Ophthalmol 1985;103:1796-806.

17 Elman MJ, Bressler NM, Qin H, et al. Expanded 2-year follow-up of ranibizumab plus prompt or deferred laser or triamcinolone plus prompt laser for diabetic macular edema. Ophthalmology 2011;118:609-14.

18 Bansal AS, Khurana RN, Wieland MR, et al. Influence of glycosylated hemoglobin on the efficacy of ranibizumab for diabetic macular edema: a post hoc analysis of the RIDE/RISE trials. Ophthalmology 2015;122:1573-9.

19 Singh RP, Habbu K, Ehlers JP, et al. The impact of systemic factors on clinical response to ranibizumab for diabetic macular edema. Ophthalmology 2016;123:1581-7.

20 Stratton IM, Adler Al, Neil HA, et al. Association of glycaemia with macrovascular and microvascular complications of type 2 diabetes (UKPDS 35): prospective observational study. BMJ 2000;321:405-12. 